\title{
A New Approach to Improve the Hemodynamic Assessment of Cardiac Function Independent of Respiratory Influence
}

Leslie Ogilvie

University of Guelph

Brittany A. Edgett

University of Guelph

Simon Gray

Cambridge Electronic Design Limited

Sally Al-Mufty

University of Guelph

Jason S. Huber

University of Guelph

Keith R. Brunt

Dalhousie Medicine New Brunswick

Jeremy A. Simpson ( $\nabla$ jeremys@uoguelph.ca )

University of Guelph

\section{Research Article}

Keywords: contractility, relaxation, heart failure, respiratory failure, ventilation

Posted Date: April 12th, 2021

DOl: https://doi.org/10.21203/rs.3.rs-382837/v1

License: (c) (1) This work is licensed under a Creative Commons Attribution 4.0 International License. Read Full License 


\section{Abstract}

Cardiovascular and respiratory systems are anatomically and functionally linked; inspiration produces negative intrathoracic pressures that act on the heart and alter cardiac function. Inspiratory pressures increase with heart failure and can exceed the magnitude of ventricular pressure during diastole. Accordingly, respiratory pressures may be a confounding factor to assessing cardiac function. While the interaction between respiration and the heart is well characterized, the extent to which systolic and diastolic indices are affected by inspiration is unknown. Our objective was to understand how inspiratory pressure affects the hemodynamic assessment of cardiac function. To do this, we developed custom software to assess and separate indices of systolic and diastolic function into inspiratory, early expiratory, and late expiratory phases of respiration. We then compared cardiac parameters during normal breathing and with various respiratory loads. Variations in inspiratory pressure had a small impact on systolic pressure and function. Conversely, diastolic pressure strongly correlated with negative inspiratory pressure. Cardiac pressures were less affected by respiration during expiration; late expiration was the most stable respiratory phase. In conclusion, inspiration is a large confounding influence on diastolic pressure, but minimally affects systolic pressure. Performing cardiac hemodynamic analysis by accounting for respiratory phase yields more accuracy and analytic confidence to the assessment of diastolic function.

\section{Highlights}

- Left ventricle pressure was predicted by intrathoracic pressure during inspiration, but not late expiration

- Diastolic parameters were more sensitive to inspiratory pressure than systolic parameters

- Parameters of cardiac pressure were more affected by respiratory phase and load than parameters of contraction or relaxation rate

- Respiratory loading increased variance in cardiac parameters

- Evaluating hemodynamics during late expiration improves assessment of cardiac function over the traditional approach

\section{Introduction}

Heart failure is the result of structural and functional changes in the myocardium that lead to impairments in ventricular filling and/or ejection of blood. ${ }^{1}$ The measurement of ventricular hemodynamics is essential for understanding changes in cardiac physiology that occur in response to experimentally induced pathologies and therapeutic interventions. ${ }^{2}$ Direct hemodynamic measurements provide a comprehensive assessment of systolic and diastolic function in experimental models of heart disease. Owing to the tight anatomical and functional relationship between the cardiovascular and respiratory systems, their relationship alters cardiac hemodynamics uniquely. Decreases in intrathoracic pressure during inspiration reduce ventricular pressure and augment venous return. ${ }^{3-5}$ Respiratory 
pressures can even exceed the magnitude of diastolic filling pressure. ${ }^{6,7}$ Thus, intrathoracic pressure could independently influence the measures of diastolic and systolic hemodynamics. While respiratory rhythms are known to influence ventricular filling and ejection volumes $3,5,8-11$, the extent to which inspiratory pressures affect the evaluation of diastolic and systolic hemodynamics is unknown.

Intrathoracic pressures oscillate between inspiratory and expiratory phases. Many acute stresses and chronic disease states increase the amplitude of intrathoracic pressure swings throughout respiration. This can occur as a result of obstruction in the airways (e.g., pulmonary edema, inflammation), diaphragmatic weakness, or elevated end-expiratory pressures. ${ }^{6,7,12,13}$ These changes in intrathoracic pressure change afterload and venous return ${ }^{14}$, impacting cardiac hemodynamics without changing the intrinsic function of the myocardium per se. Experimental variables that impact respiratory function (e.g., myocardial infarction, pressure-overload heart failure, metabolic impairments) $)^{7,15}$ could cause indirect changes in cardiac function that lead to misinterpretation.

Here we aimed to understand how inspiratory pressure influences hemodynamic indices of diastolic and systolic function. To test this, we simultaneously measured left ventricle (LV) pressure and tracheal pressure during eupnea and with increasing severities of respiratory resistance loads to mimic changes in respiratory function expected in animal models of heart failure. Using custom software, diastolic and systolic parameters were then separated into inspiratory, early expiratory, and late expiratory phases of respiration for comparable analysis. We show that diastolic pressure and to a lesser extent, systolic pressure, was predicted by tracheal pressure during inspiration. Conversely, tracheal pressure during late expiration showed no correlation with cardiac pressure, providing an assessment of cardiac function independent of respiratory pressure. Diastolic parameters showed a large percent change from expiratory to inspiratory pressure, while systolic parameters changed minimally. Additionally, respiratory resistance loads increased variability in all cardiac parameters, which was more pronounced in the diastolic parameters. Together our data indicate that respiration is a confounding influence to the evaluation of diastolic function. We show a new approach to remove this influence from hemodynamic analyses to provides a more rigorous assessment of diastolic and systolic function.

\section{Results}

\section{Respiratory resistance loads alter respiratory function}

We assessed cardiac hemodynamics by invasive catheterization while simultaneously recording tracheal pressure to first understand the influence of resistance loading on respiratory function (Figure 1). As expected, mild resistance decreased peak inspiratory tracheal pressure compared to eupnea and decreased even further with moderate airway resistance (Table 1). Although not significant $(p=0.053)$, there was a $10 \%$ increase in respiratory rate from eupnea with added respiratory loads (Table 1 ). Respiration can alter cardiac function by either changing the magnitude of intrathoracic pressure or duration of respiratory phase. Thus, we determined the number of cardiac cycles per respiratory phase with increasing respiratory resistance load (Figure 2A). During eupnea, late expiration had more cardiac 
cycles compared to inspiration and early expiration, which were equivalent (Figure 2B, Table 2). Resistance loading altered the number of cardiac cycles that occurred during all respiratory phases. Specifically, mild and moderate resistance similarly increased the number of inspiratory cardiac cycles compared to eupnea by decreasing the number of cycles during expiration. Since the number of cardiac cycles is representative of time, our data indicate that respiratory loading increased inspiratory time and decreased expiratory time compared to eupnea. Together, these data demonstrate that respiratory loads altered the duration of the respiratory phases, which may enhance phase-specific effects of respiration on cardiac function.

\section{Respiratory phase and resistance loads affect cardiac function}

To identify how respiratory pressure influences cardiac function, we analyzed systolic and diastolic parameters during inspiratory, early expiratory, and late expiratory phases, and with respiratory phases combined during eupnea and added resistance loads (Figure 3, S1; DOI: 10.6084/m9.figshare.13022759).

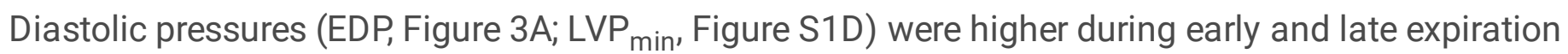
compared to inspiration, regardless of respiratory load. The same occurred when comparing EDP and $L V P_{\text {min }}$ during early and late expiration to the combined phases, except for EDP at early expiration during eupnea. This indicates that inspiratory-mediated decreases in diastolic pressure drive the observed differences between the combined group and expiratory phases. Interestingly, diastolic pressures were affected by respiratory loading only during inspiration, decreasing as the load increased. This was similar for combined phases due to the negative influence of inspiratory pressure. Diastolic pressures were consistent between early and late expiration across resistance loads, aside from a slight increase during early expiration with moderate resistance. As such, inspiration decreased diastolic pressures, while expiration provided a stable assessment of diastolic pressure, with minimal influence from respiration.

Peak relaxation rate (i.e., $\mathrm{dP} / \mathrm{dt}_{\mathrm{min}}$ ) was unaffected by respiratory load (Figure 3B). During eupnea, $\mathrm{dP} / \mathrm{dt}_{\min }$ was lower during late expiration compared to the combined phases (Figure 3B). The only additional changes observed were with moderate loading, where $\mathrm{dP} / \mathrm{dt}_{\min }$ was higher during inspiration compared to all other respiratory phases and when the phases were combined. Altogether, peak relaxation rate was resistant to influences from respiratory load, with only small changes observed across respiratory phases. The relaxation time constant, Tau (Weiss, Glantz, Logistic), is an index of ventricular relaxation rate. Similar to $\mathrm{dP} / \mathrm{dt}_{\mathrm{min}}$, Tau was not affected by respiratory load (Figure S1A, B and C). Tau Logistic was modestly influenced by respiratory phase, while Tau Weiss and Glantz were not. Thus, relaxation rate was less susceptible to changes in respiratory pressure and phase.

Systolic pressure (i.e., peak LVP) decreased during early expiration compared to all other respiratory phases and when the phases were combined in all respiratory load conditions, except compared to inspiration during moderate resistance (Figure $3 \mathrm{C}$ ). Conversely, systolic pressure increased during late expiration compared to all other phases and the combined phases for all loads, excluding during eupnea, where late expiratory and inspiratory systolic pressure were similar. Systolic pressure was affected by resistance load, decreasing with any added load compared to eupnea but with no difference between 
mild and moderate loads themselves. This occurred for inspiration, early expiration and when the respiratory phases were combined. Late expiratory systolic pressure was the phase least influenced by resistance load, as there was no difference in peak LVP during moderate resistance compared to eupnea or mild load. This is likely because late expiration is the only respiratory phase where intrathoracic pressures are positive throughout.

Respiration had minimal effect on peak contraction rate (i.e., $\mathrm{dP} / \mathrm{dt}_{\max }$ ); only late expiration was slightly higher than the other phases during eupnea (Figure 3D). Contraction rate at LVP of $40 \mathrm{mmHg}$ (i.e., $\mathrm{dP} / \mathrm{dt}_{@ L L P 40}$ ) was calculated to provide an afterload-independent index of contractility since $\mathrm{dP} / \mathrm{dt}_{\max }$ is sensitive to preload and afterload. Contractility, independent of afterload, was not different between the respiratory phases during eupnea (Figure S1E). Mild and moderate respiratory loading for $\mathrm{dP} / \mathrm{dt}_{@ L L P 40}$ was higher during early expiration compared to all other phases and when the phases were combined. Conversely, inspiration decreased $\mathrm{dP} / \mathrm{dt}_{@ L \mathrm{LV} 40}$ during mild and moderate respiratory loads compared to the combined phases and early expiration. These data support a biphasic pattern of contraction rate throughout respiration, where large inspiratory pressures impede contractility and expiratory pressures augment contractility during conditions with additional respiratory load. Surprisingly, although $\mathrm{dP} / \mathrm{dt}_{@ \mathrm{LLP} 40}$ is less sensitive to changes in ventricle loading than $\mathrm{dP} / \mathrm{dt}_{\text {max }}$, our data show that $\mathrm{dP} / \mathrm{dt}_{@ L V P 40}$ is more sensitive to respiratory influences. Lastly, heart rate was also generally unaffected by respiratory function, with only a slight increase during inspiration compared to the combined phases with moderate resistance (Figure S1F). Altogether, our data indicate that respiratory phases and loads elicited changes in cardiac function that were specific to each systolic and diastolic parameter. Respiration had a greater effect on parameters of cardiac pressure, rather than parameters reflecting contraction or relaxation rate. Of the cardiac parameters most affected by intrathoracic pressure (e.g., EDP, LVP ${ }_{\min }$, peak LVP), evaluating data during late expiration minimized aberrations.

\section{EDP is predicted by tracheal pressure during inspiration, but not during late expiration}

We performed linear regressions to determine whether respiratory pressure correlates to diastolic and systolic pressure. Analyses were limited to inspiration and late expiration as they have the least and most stable tracheal pressures during respiration, respectively. During inspiration, diastolic pressure decreased as tracheal pressure decreased (Figure 4A); $64 \%$ of the variance observed in EDP was due to changes in tracheal pressure. Conversely, diastolic pressure was unaffected by tracheal pressure during late expiration (Figure 4B). Systolic pressure also decreased as tracheal pressure decreased during inspiration, with $27 \%$ of the variance in peak LVP explained by changes in tracheal pressure (Figure 4C). During late expiration, systolic pressure was not influenced by tracheal pressure (Figure 4D). Thus, inspiratory tracheal pressure was a stronger predictor of diastolic compared to systolic pressure, suggesting that diastolic function is more influenced by inspiration than systolic function. In contrast to the large influence of inspiration on cardiac pressure, late expiration had no effect. Therefore, evaluating cardiac function during the late expiratory phase provides a method to assess diastolic and systolic function independent of respiration. 
Variability in data collection determines confidence or influences power calculation assumptions that pose ethical and economic impacts on study design. Variability in hemodynamic parameters would increase the demand on sample size. Thus, we determined whether respiratory resistance loads or phases affect variance in diastolic and systolic parameters (Figure S2; DOI: d10.6084/m9.figshare.13022759; Table S1-S4; DOI: $10.6084 / \mathrm{m}$.figshare.13022813). With respect to eupneic load, both mild and moderate resistance loads proportionately increased variance by a similar amount in diastolic pressures (EDP, Figure $5 A ; L_{\text {min }}$, Figure S2D). The addition of a load not the severity of the load itself, impacts variance in diastolic pressure. Load also increased variance in $\mathrm{dp} / \mathrm{dt}_{\mathrm{min}}$ yet, in this parameter the severity of load (not just the addition of it, Figure 5B) contributed to greater variance. How Tau is measured determines its susceptibility to variance both from an intragroup and intergroup perspective relative to eupnea (Figure S2). Tau Logistic shows the least variability in any measure, with any load. Tau Weiss has high intragroup variability, which leads to a false expectation of no significant intergroup variance (Figure S2A). Tau Glantz shows a susceptibility to increased load for elevated intragroup variability and significantly increased intergroup variance (Figure S2B). The superiority of Tau Logistic is clear, and yet also has significant elevated variance with respect to increasing resistance load, reflecting an impact to accuracy despite superior precision. Respiratory load increases Tau Logistic variance with the severity of load (not simply the addition of it, Figure S2C). With regard to variance in all diastolic parameters, there was no indication that a specific respiratory phase was driving the variance per se. Tau Logistic is a superior index to evaluate Tau for its precision and-when respiration is accounted for-accuracy over other Tau indices.

With respect to eupnea, resistance loads increased variance in systolic pressure, which was proportional to the severity of load (Figure $5 \mathrm{C}$ ). Moderate loading also increased the variance in $\mathrm{dP} / \mathrm{dt}_{\text {max }}$ compared to eupnea or mild load (Figure 5D). Variance in $\mathrm{dP} / \mathrm{dt}_{@ L V P 40}$ was increased with the addition of a resistance load, showing no difference between the loads themselves (Figure S2E). Moderate loading increased variance in heart rate compared eupnea (Figure S2F). While there was little evidence supporting a change in variance during a specific respiratory phase, resistance loading increased variability for all diastolic and systolic parameters.

\section{Diastolic parameters show greater variance than systolic parameters}

To compare variability between diastolic and systolic function, we calculated coefficients of variation for each cardiac parameter across the respiratory phases and loads (Table S5; DOI: 10.6084/m9.figshare.13022813). During eupnea and both resistance loads, diastolic parameters (EDP, Tau Weiss, and $\mathrm{LVP}_{\min }$ ) had the largest variability of all cardiac parameters measured, regardless of respiratory load. Consistent with the observation of large intragroup variability, Tau Weiss had greater variation coefficients than Tau Glantz or Tau Logistic in all respiratory conditions. This is likely because Tau Weiss does not account for changes in the minimum LV pressure asymptote (e.g., due to intrathoracic pressure changes throughout respiration that vertically shift the ventricular relaxation 
curve). Conversely, Tau Glantz and Logistic consider these pressure changes when the ventricle is fully relaxed and were thus less affected by respiratory influence $(C V<15 \%)$. Systolic pressure (i.e., peak LVP) had the smallest variability compared to all other parameters. Similarly, coefficients of variation for most rate parameters (i.e., $\mathrm{dP} / \mathrm{dt}_{\text {max }}, \mathrm{dP} / \mathrm{dt}_{@ L L V 40}, \mathrm{dP} / \mathrm{dt}_{\text {min }}$, and heart rate) were also small $(\mathrm{CV}<12 \%)$ during eupnea and with mild resistance. Together, these data indicate that variability of diastolic parameters is more sensitive than systolic parameters, regardless of intrathoracic pressure.

Diastolic pressures have the highest magnitude of change from late expiration to inspiration in all cardiac parameters

We determined the percent change of systolic and diastolic parameters from late expiration to inspiration (Figure 6). All systolic parameters showed less than $5 \%$ change in all respiratory conditions. Conversely, diastolic pressures $\left(E D P, L V P_{\text {min }}\right.$ ) had the largest change of all parameters from late expiration to inspiration during eupneic breathing, which was further amplified with resistance loading. This is likely because diastolic pressures are very low compared to systolic pressures (i.e., $\sim 5 \mathrm{mmHg}$ during diastole versus $\sim 120 \mathrm{mmHg}$ during systole). Therefore, small changes in ventricular pressure (e.g., due to inspiratory influences) have a larger effect on diastolic pressure. Tau Weiss showed a greater percent change compared to Tau Glantz and Tau Logistic. Respiratory function is an important consideration when evaluating diastolic pressure because of the sensitivity of diastolic function to inspiration and the potential for misinterpretations in hemodynamic data when respiratory influences are not considered.

\section{Discussion}

Respiration facilitates a rhythmic influence on cardiac physiology. $3,5,16$ Accordingly, cardiac pressures and volumes are altered by changes of intrathoracic pressure throughout respiration. To our knowledge, this is the first study to concurrently measure intrathoracic pressures and cardiac hemodynamics to stratify parameters of systolic and diastolic function by respiratory phase. Additionally, we used respiratory resistance loads to mimic inspiratory pressures expected in experimental models of heart failure, comparing the effects of respiration on cardiac function during normal and resisted breathing. Our salient findings are as follows: 1 ) inspiratory pressure was a strong predictor of end diastolic pressure and a weak predictor of peak systolic pressure, showing that diastolic function is more sensitive to inspiration than systolic function, 2) cardiac parameters assessed during late expiration were the least affected by changes in tracheal pressure, providing a means to evaluate cardiac function with minimal respiratory influence, 3 ) respiratory loading increased the variability in all cardiac parameters, which was most pronounced in the diastolic parameters; experimental models with altered respiratory function likely require a greater number of animals to detect group differences, 4) based on our data, researchers should control respiratory rate and depth, and level of anesthesia when evaluating cardiac function, as these alter respiratory function, which in turn influence cardiac function.

Previous literature has shown that respiration produces temporally distinct direct and indirect effects on the heart ${ }^{3}$ (Figure 7). Decreases in intrathoracic pressure during inspiration reduce ventricular afterload 
causing a decline in intraventricular pressure. Additionally, the pressure gradient between the thoracic and abdominal cavities during inspiration augments venous return to the right atrium, increasing right ventricle preload and stroke volume. Simultaneously, as right ventricle filling increases, this causes septal shifting - called ventricular interdependence - which transiently decreases left ventricle filling and stroke volume. $^{3,17}$ These effects on the left ventricle, secondary to an increase in right ventricle filling, represent the immediate and indirect effects of inspiration. In subsequent cardiac beats, which corresponds with the expiratory phase of respiration, increased right ventricle output increases left ventricle filling and stroke volume ${ }^{17}$; this is the delayed effect of inspiration on left ventricle function. Our data are in agreement with existing literature defining the influence of respiration on cardiac function. ${ }^{3-6,9,11-14,17-20}$ We also show that end diastolic pressure, and to a lesser extent, systolic pressure is predicted by inspiratory pressure. As intrathoracic pressure declines during inspiration and is transmitted across the ventricle wall, there is a resulting decrease in intraventricular pressure. Since intraventricular pressure

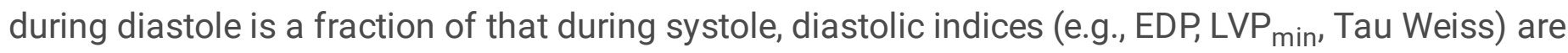
more sensitive to inspiration than systolic indices. Systolic pressure also decreases during inspiration, however, the effect was minimal compared to diastolic pressure and only observed with an added respiratory load. These findings are critical to our assessment and interpretation of diastolic function with a hemodynamic approach, as alterations in respiratory function may present confounding effects on diastolic pressure without directly changing myocardial function. Interestingly, parameters of cardiac pressure were more influenced by respiratory phase and load than parameters of contraction or relaxation rate. A possible explanation for this observation is that rate parameters exhibited large variability, which may have prevented our ability to detect value differences across the respiratory phases and loads. Alternatively, this finding may suggest that parameters of contraction or relaxation rate are more representative of intrinsic properties of myocardial function (e.g., myofilaments, elastance) and are less affected by external influences, such as intrathoracic pressure.

Research in diastolic dysfunction has surged over the last decade, yet no effective therapies exist to improve myocardial relaxation and/or filling. Elevated end diastolic pressure is recognized as a key contributor to the development of heart failure. ${ }^{21-23}$ Indeed, the gold standard for diagnosing heart failure with preserved ejection fraction-characterized by left ventricular diastolic dysfunction with normal systolic function-is elevated end diastolic pressure by cardiac catheterization. ${ }^{24}$ Thus, there is a need to comprehensively and accurately evaluate diastolic function to improve our understanding of the pathophysiology of diastolic dysfunction, which could ultimately lead to the development of more effective therapeutics for heart failure by unmasking the definitive functional features with greater precision and accuracy inherent to the fundamental cause. To improve accuracy in hemodynamic data analysis, we suggest reporting diastolic parameters during the late expiratory phase of respiration when respiratory function is not equivalent between experimental groups. This analysis will minimize the confounding effects of respiration on the interpretation of the features of diastolic function.

In experimental models of heart failure (e.g., pressure-overload heart failure, myocardial infarction, hypoxia) alterations in respiratory function are expected. ${ }^{7,25,26}$ We used mild and moderate resistance 
loads to mimic the inspiratory intrathoracic pressures observed in various cardiac and respiratory pathologies (Figure 8). Further, agents used to induce and maintain general anesthesia have respiratory depressant effects, which may, in turn, affect cardiac function. ${ }^{27,28}$ Alterations in respiratory function, due to anesthetic type/level or experimental intervention, may yield data that does not represent true cardiac function or produces increased intragroup variability. Thus, our findings highlight the importance of taking respiratory rate and depth, and anesthetic level into account when interpreting hemodynamic data.

In the present study, we show that respiratory resistance loads increase variance in all cardiac parameters. Heightened respiratory influences may increase the sample size required to detect differences in hemodynamic function between experimental groups. Although separating cardiac parameters by respiratory phase minimized the influence of inspiration on ventricular pressure, there was no one respiratory phase that showed a consistently reduced variability during eupneic breathing or with respiratory loading. This finding is likely because animals did not all respond the same way to respiratory loading. For example, while some animals showed increased inotropy (contractility) and lusitropy (relaxation rate) with resistance loading, others showed major decreases. These divergent responses greatly increased the variability in cardiac parameters as resistance load increased, hindering our ability to detect subtle changes in variance between the respiratory phases.

\section{Study Limitations}

We performed our analysis with a focus on left ventricle function. The low systolic and diastolic pressures in the right ventricle make it more susceptible to inspiratory influences and changes in afterload than the left ventricle. ${ }^{16}$ Future studies should investigate indices of right ventricle function, separated by respiratory phase, to understand how respiration affects the assessment of right ventricle hemodynamics. Secondly, this study was performed in rats, therefore, whether our observations are conserved in mice remains to be investigated. We anticipate that our findings in rats will translate to mouse models since mice also produce large intrathoracic pressure swings throughout respiration. ${ }^{7}$ Lastly, this work was performed in male animals only. Future studies could investigate how respiration influences cardiac hemodynamics in females, taking into account the effects of female estrous cycling and hormonal influences on cardiac and respiratory physiology. ${ }^{29,30}$ However, despite a small change in size, the structural anatomy is sex consistent and should not be fundamentally different to the conclusions we report here.

\section{Conclusion}

Together, our data show that respiration is a confounding influence on the assessment of cardiac hemodynamics. While inspiratory pressures have a slight effect on systolic function, the effect on diastolic function is profound. Given the ongoing interest in characterizing diastolic dysfunction and its contribution to all forms of heart failure, accurate methods for measuring diastolic function are critical. We propose that in scenarios where respiratory function is not equivalent between experimental groups, 
evaluating hemodynamic parameters during the late expiratory phase of respiration provides the most accurate, respiratory-independent assessment of cardiac physiology. In doing so, we will minimize the confounding influence of inspiration on systolic and diastolic function and limit misinterpretations or failure to identify salient small effects within hemodynamic data that would be pathophysiologically relevant to the mechanism underlying feature changes. This will be important for data reproducibility as research in experimental models of heart failure continues to uncover important insights in disease progression and therapeutic targets for improving cardiac health.

\section{Methods}

Ethical approval. Experiments, approved by the Animal Care Committee of Queen's University and in conformity with the Canadian Council on Animal Care guidelines, were conducted on pentobarbital sodium-anesthetized, male Sprague-Dawley rats (300-460 g; $65 \mathrm{mg} / \mathrm{kg}$ I.P.), supplemented as required to prevent a pedal reflex. This study was carried out in compliance with the ARRIVE guidelines. ${ }^{31}$

Assessment of cardiorespiratory function. In brief, after a surgical plane of anesthesia was established, rats $(n=7)$ were placed supine. Body temperature was maintained at $37.5^{\circ} \mathrm{C}$ with a servo-controlled heating pad. One port of a tracheal cannula was connected to a pressure transducer to measure tracheal pressure (used as a surrogate for intrathoracic pressure to identify the beginning and end of each respiratory phase); the other port was attached to a two-way valve (Hans Rudolf 2300, Kansas City, MO, USA). The right carotid artery was isolated, and a pressure catheter was inserted and advanced into the left ventricle. Once physiological pressures were recorded during eupneic breathing, a small clamp was attached to the inspiratory side of the two-way valve and was fully constricted to produce the maximum inspiratory pressure for each animal. To produce inspirations deeper than eupneic breathing and load the respiratory system, the clamp was partially tightened to generate mild ( 20-30\% of the maximum inspiratory pressure) or moderate ( $\sim 40-50 \%$ of the maximum inspiratory pressure) resistance loads. Left ventricle function was recorded at a sampling rate of $1 \mathrm{kHz}$, then rats were euthanized by pentobarbital overdose. Parameters of left ventricle function (i.e., heart rate; peak left ventricle pressure, peak LVP; end diastolic pressure, EDP; minimum left ventricle pressure, $\mathrm{LVP}_{\text {min }}$; peak contraction and relaxation rates, $\mathrm{dP} / \mathrm{dt}_{\max }$ and $\mathrm{dP} / \mathrm{dt}_{\mathrm{min}}$, respectively; rate of change of pressure at left ventricle pressure of $40 \mathrm{mmHg}$, $\mathrm{dP} / \mathrm{dt}_{@ L L P 40} ;$ Tau Weiss; Tau Glantz; Tau Logistic) were analyzed with custom analysis using Spike2 version 10.01 software (CED Spike2, Cambridge, UK). For each respiratory intervention (i.e., eupnea, mild and moderate resistance loads), a 10-second interval of data was obtained from a region of stable left ventricle pressure and parameters were analyzed without separation by respiratory phase (combined), then separated into inspiratory, early expiratory, and late expiratory phases of respiration for further analysis. In cases where a cardiac cycle fell across two respiratory phases, the cycle was placed into the respiratory phase with the majority (>50\%) of data points for that given cardiac cycle. Tau Weiss, Tau Glantz, and Tau Logistic were calculated as described previously. ${ }^{32}$ 
Data analyses. Statistical analyses were conducted with SPSS v. 27 (IBM Corp., Armonk, NY, USA). For each cardiac parameter measured, separate 3 (intervention: eupnea, mild resistance, moderate resistance) $\times 4$ (phase: combined, inspiration, early expiration, late expiration) within-subject analyses of variance (ANOVAs) were conducted. Mauchly's Test of Sphericity was used to determine whether the variances of the differences between conditions were equal. If significant, the Greenhouse-Geisser correction was used where appropriate. One-way repeated-measures ANOVAs were used to further investigate significant interactions and main effects. A Sidak correction was used for all pairwise comparisons. Linear regression was performed to describe how tracheal pressure affects diastolic and systolic pressure during inspiratory and late expiratory phases. For linear regression, data were analyzed only during inspiratory and late expiratory phases since inspiration generates the largest swings in tracheal pressure and tracheal pressure changes minimally during late expiration. For these tests, statistical significance was determined at $p<0.05$. The variability of all cardiac parameters was assessed by calculating within-subject coefficients of variation (CV) and, where Mauchly's Test was significant, one-tailed Pearson's correlation coefficients were performed. When normality could not be assumed, Spearman's correlation coefficient was used instead. As these were pair-sampled, they should positively correlate; differences in subject response and thus group variability were determined to be present when $p>0.05$. Graphs were made using Prism 8 (GraphPad Software Inc., San Diego, CA, USA). All results are expressed as means \pm standard deviation (SD) unless otherwise indicated.

\section{Declarations}

\section{ACKNOWLEDGEMENTS}

We kindly thank S. Iscoe for helpful discussions. Figure 7 created with BioRender.com.

\section{GRANTS}

This study was supported by Natural Sciences and Engineering Research Council, Canadian Institutes of Health Research, and Heart and Stroke Foundation of Canada grants (to J. A. Simpson and K. R. Brunt) and Killam Foundation grant (to B. A. Edgett).

\section{DISCLOSURES}

No conflicts of interest, financial or otherwise, are declared by the authors.

\section{AUTHOR CONTRIBUTIONS}

LMO, BAE, SG, KRB, JAS conceived and designed research, LMO, BAE, SG, JSH, JAS analyzed data, LMO, $B A E, S G, J S H, K R B$, JAS interpreted results of experiments, LMO, BAE, KRB, JAS prepared figures, LMO, $B A E$, SA, KRB, JAS drafted manuscript, LMO, BAE, SA, JSH, KRB, JAS edited and revised manuscript, LMO, $B A E, S G, S A, J S H, K R B, J A S$ approved final version of manuscript. 


\section{References}

1. Yancy, C. W. et al. Guidelines in review: 2013 ACCF/AHA guideline for the management of heart failure: a report of the American College of Cardiology Foundation/American Heart Association Task Force on Practice Guidelines. Circulation128, e240-3327 (2013).

2. Lindsey, M., Kassiri, Z., Virag, J., de Castro Bras, L. \& Scherrer-Crosbie, M. Guidelines for measuring cardiac physiology in mice. Am J Physiol Heart Circ Physio/314, H733-H752 (2018).

3. Amoore, J. N. \& Santamore, W. P. Model studies of the contribution of ventricular interdependence to the transient changes in ventricular function with respiratory efforts. Cardiovasc Res23, 683-694 (1989).

4. Olsen, C. O., Tyson, G. S., Maier, G. W., Davis, J. W. \& Rankin, J. S. Diminished stroke volume during inspiration: A reverse thoracic pump. Circulation72, 668-679 (1985).

5. Scharf, S. M., Brown, R., Saunders, N. \& Green, L. H. Effects of normal and loaded spontaneous inspiration on cardiovascular function. J Appl Physiol Respirat Environ Exercise Physio/47, 582-590 (1979).

6. Simpson, J. A., Brunt, K. R. \& Iscoe, S. Repeated inspiratory occlusions acutely impair myocardial function in rats. J Physio/586, 2345-2355 (2008).

7. Foster, A. J. et al. Central-acting therapeutics alleviate respiratory weakness caused by heart failureinduced ventilatory overdrive. Sci Trans/ Med9, 1-16 (2017).

8. Kim, B. H. et al. Effects of spontaneous respiration on right and left ventricular function: Evaluation by respiratory and ECG gated radionuclide ventriculography. J Nucl Med28, 173-177 (1987).

9. Scharf, S. M. The effect of decreased intrathoracic pressure on ventricular function. J Sleep Res4, 53-58 (1995).

10. Scharf, S. M., Bianco, J. A., Tow, D. E. \& Brown, R. The effects of large negative intrathoracic pressure on left ventricular function in patients with coronary artery disease. Circulation63, 871-875 (1981).

11. Innes, J., de Cort, S., Kox, W. \& Guz, A. Within-breath modulation of left ventricular function during normal breathing and positive-pressure ventilation in man. Journal of Physiology460, 487-502 (1993).

12. Simpson, J. A. \& Iscoe, S. Cardiorespiratory failure in rat induced by severe inspiratory resistive loading. J Appl Physio/102, 1556-1564 (2007).

13. Simpson, J. A., Brunt, K. R., Collier, C. P. \& Iscoe, S. Hyperinflation-induced cardiorespiratory failure in rats. J Appl Physio/107, 275-282 (2009).

14. Scharf, S. M. Cardiovascular effects of airways obstruction. Lung169, 1-23 (1991).

15. Gray, B. A., Hyde, R. W., Hodges, M. \& Yu, P. N. Alterations in lung volume and pulmonary function in relation to hemodynamic changes in acute myocardial infarction. Circulation59, 551-559 (1979).

16. Pueschner, A. et al. The prevalence, correlates, and impact on cardiac mortality of right ventricular dysfunction in nonischemic cardiomyopathy. JACC: Cardiovasc Imaging10, 1225-1236 (2017). 
17. Scharf, S. M., Graver, L. M., Khilnani, S. \& Balaban, K. Respiratory phasic effects of inspiratory loading on left ventricular hemodynamics in vagotomized dogs. J Appl Physiol73, 995-1003 (1992).

18. Brecher, G. A. \& Hubay, C. A. Pulmonary blood flow and venous return during spontaneous respiration. Circulation research111, 210-214 (1955).

19. Aliverti, A. et al. Concomitant ventilatory and circulatory functions of the diaphragm and abdominal muscles. Journal of Applied Physiology109, 1432-1440 (2010).

20. Cheyne, W. S., Harper, M. I., Gelinas, J. C., Sasso, J. P. \& Eves, N. D. Mechanical cardiopulmonary interactions during exercise in health and disease. J Appl Physio/128, 1271-1279 (2020).

21. Watanabe, S. et al. Myocardial stiffness is an important determinant of the plasma brain natriuretic peptide concentration in patients with both diastolic and systolic heart failure. Eur Heart $\mathbf{J 2 7}, 832-$ 838 (2006).

22. Mielniczuk, L. M. et al. Left ventricular end-diastolic pressure and risk of subsequent heart failure in patients following an acute myocardial infarction. Congest Heart Fail13, 209-214 (2007).

23. Zile, M. R., Baicu, C. F. \& Gaasch, W. H. Diastolic heart failure - abnormalities in active relaxation and passive stiffness of the left ventricle. New Engl J Med350, 1953-1959 (2004).

24. Paulus, W. J. et al. How to diagnose diastolic heart failure: A consensus statement on the diagnosis of heart failure with normal left ventricular ejection fraction by the Heart Failure and Echocardiography Associations of the European Society of Cardiology. Eur Heart J28, 2539-2550 (2007).

25. Kazemi, H., Parsons, E. F., Valenca, L. M. \& Strieder, D. J. Distribution of pulmonary blood flow after myocardial ischemia and infarction. Circulation41, 1025-1030 (1970).

26. Saaby, L. et al. Classification of myocardial infarction: Frequency and features of type 2 myocardial infarction. Am J Med126, 789-797 (2013).

27. Dahan, A. \& Teppema, L. J. Influence of anesthesia and analgesia on the control of breathing. $\mathrm{Br} J$ Anaesth91, 40-49 (2003).

28. Janssen, B. J. A. et al. Effects of anesthetics on systemic hemodynamics in mice. Am J Physiol Heart Circ Physiol287, H1618-H1624 (2004).

29. Wolfe, L. A., Kemp, J. G., Heenan, A. P., Preston, R. J. \& Ohtake, P. J. Acid-base regulation and control of ventilation in human pregnancy. Can J Physiol Pharmacol76, 815-827 (1998).

30. Nettlefold, L., Jensen, D., Janssen, I. \& Wolfe, L. A. Ventilatory control and acid-base regulation across the menstrual cycle in oral contraceptive users. Respir Physiol Neurobio/158, 51-58 (2007).

31. Percie du Sert, N. et al. The ARRIVE guidelines 2.0: Updated guidelines for reporting animal research. Exp Physio/105, 1459-1466 (2020).

32. Ogilvie, L. M. et al. Hemodynamic assessment of diastolic function for experimental models. Am J Physiol Heart Circ Physio/318, H1139-H1158 (2020).

\section{Tables}


Table 1. Respiratory loading increases the work of breathing.

\begin{tabular}{|llll|}
\hline & Eupnea & Mild Resistance & Moderate Resistance \\
\hline $\begin{array}{l}\text { Ptr Inspiration } \\
\text { (mmHg) }\end{array}$ & $-1.4 \pm 0.9$ & $-13.5 \pm 4.1 *$ & $-21.5 \pm 4.9 * \dagger$ \\
\hline $\begin{array}{l}\text { Respiratory Rate } \\
\text { (breaths/min) }\end{array}$ & $106 \pm 21$ & $116 \pm 17$ & $117 \pm 12$ \\
\hline
\end{tabular}

Inspiratory tracheal pressure ( $\left.P \operatorname{tr}_{\text {Inspiration }}\right)$ and respiratory rate during eupnea, mild, and moderate resistance loading. Data are presented as mean $\pm S D ; * p<0.05$ vs. eupnea, $\dagger p<0.05$ mild vs. moderate resistance load. All data were analyzed using a within-subject one-way repeated measures ANOVA with Sidak's correction, $\mathrm{n}=7$.

Table 2. Number of cardiac cycles in each respiratory phase across loading interventions.

\begin{tabular}{|llll|}
\hline & Eupnea & Mild Resistance & Moderate Resistance \\
\hline Inspiration & $16 \pm 4$ & $26 \pm 5^{\mathrm{d}}$ & $27 \pm 9^{\mathrm{d}}$ \\
\hline Early Expiration & $17 \pm 6$ & $12 \pm 5^{\mathrm{b}, \mathrm{d}}$ & $15 \pm 3^{\mathrm{b}}$ \\
\hline Late Expiration & $32 \pm 7^{\mathrm{b}, \mathrm{c}}$ & $26 \pm 3^{\mathrm{c}}$ & $25 \pm 4^{\mathrm{c}}$ \\
\hline
\end{tabular}

Data represent the number of cardiac cycles that occurred during each respiratory phase with eupneic breathing and mild and moderate resistance loads. Data are presented as mean \pm SD. Two-way withinsubjects ANOVA revealed a significant interaction with a main effect of respiratory phase. b, different from inspiration within a respiratory load; $c$, different from early expiration within a respiratory load; $d$, different from eupnea within a respiratory phase; $p<0.05$ for all, $n=7$.

\section{Figures}




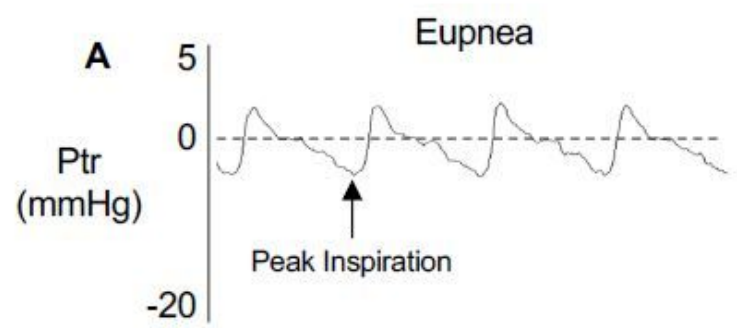
$(\mathrm{mmHg})$

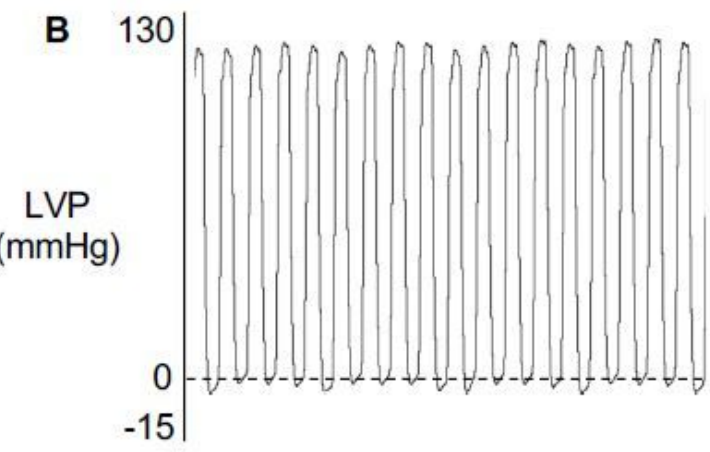

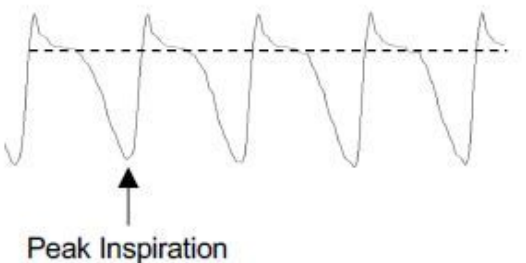

Peak Inspiration

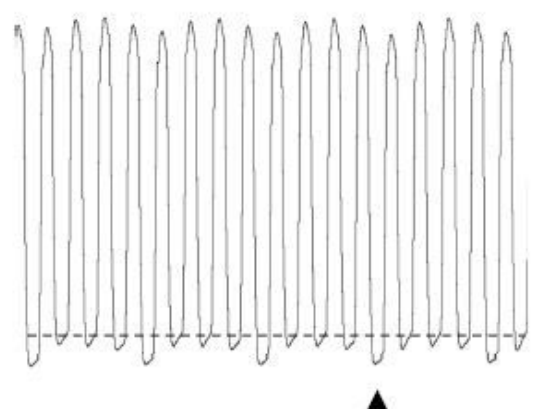

Moderate Resistance

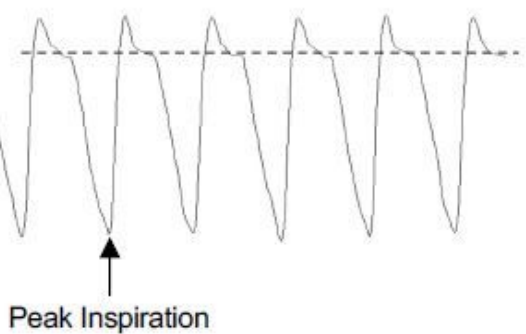

Peak Inspiration

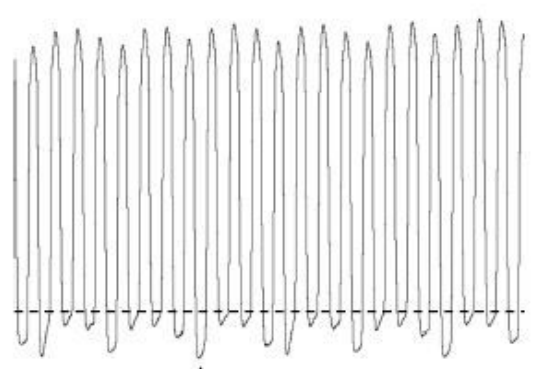

Inspiration causes more negative end diastolic pressures

\section{Figure 1}

Assessment of tracheal pressure and invasive hemodynamics. (A) Representative tracings of tracheal pressure (Ptr) (A) and left ventricle pressure (LVP) (B) during eupnea, mild, and moderate resistance loads.

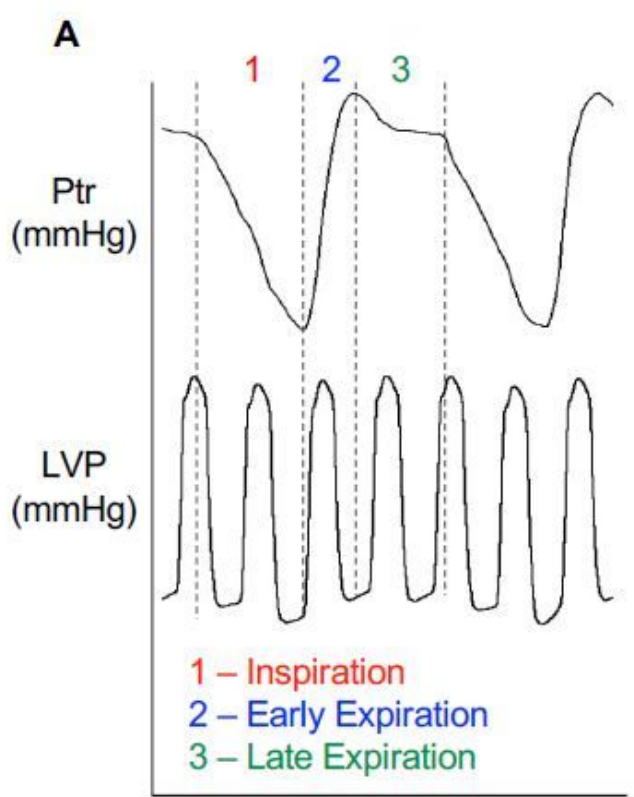

B Eupnea

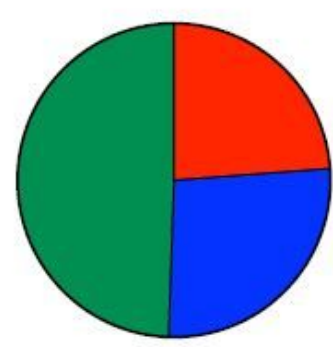

Cardiac Cycles During Inspiration

- Cardiac Cycles During Early Expiration

- Cardiac Cycles During Late Expiration

Time (s) 


\section{Figure 2}

Respiration affects the number of cardiac cycles in each respiratory phase. Representative tracing for a mild respiratory load. Inspiratory ( 1 , red), early expiratory (2, blue), and late expiratory ( 3 , green) phases of respiration were identified by tracheal pressure (Ptr) (A). Inspiration was defined between the decline in Ptr and peak negative Ptr. Early expiration was defined between peak negative and maximum Ptr. Late expiration was defined as the plateau phase between maximum Ptr and the fall in Ptr at the start of inspiration. Left ventricle pressure (LVP) was recorded simultaneously with Ptr and parameters of cardiac function were separated by respiratory phase. (B) Graphical representation of the number of cardiac cycles that occur during inspiration, early expiration, and late expiration. Data are presented as mean values, $n=7$.

- Combined - Inspiration = Early Expiration \& Late Expiration

A

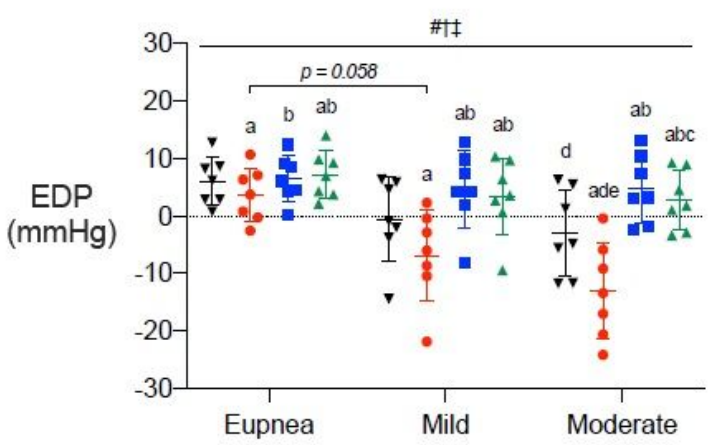

C

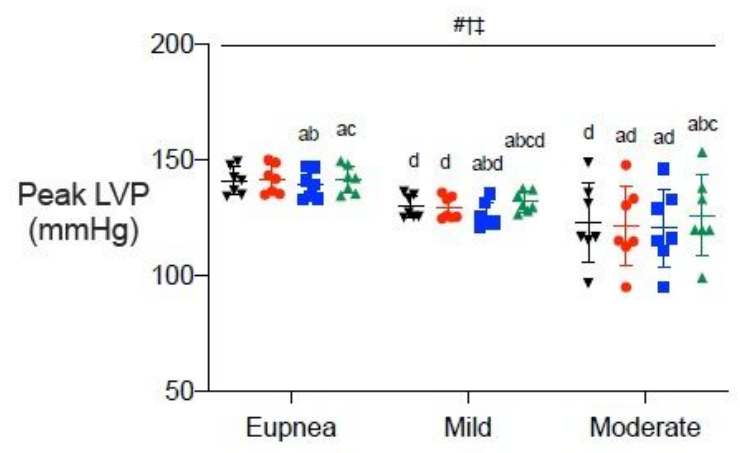

B

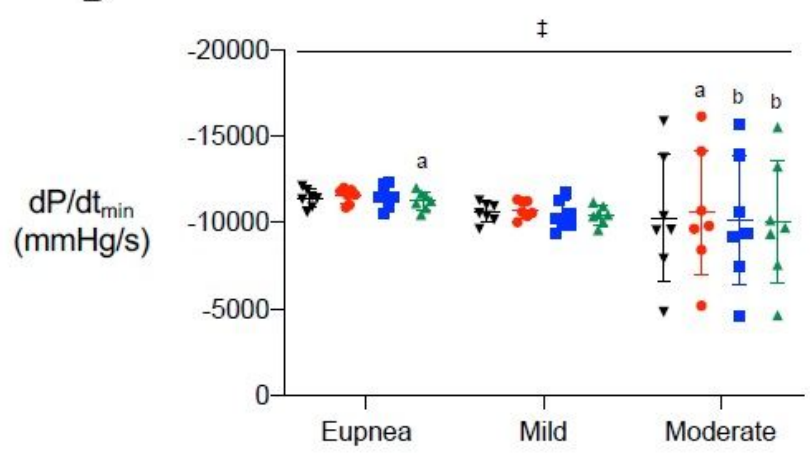

D

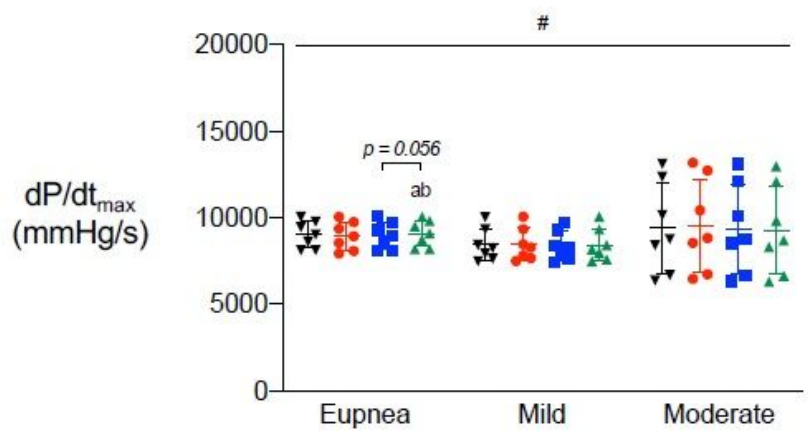

\section{Figure 3}

Cardiac function is influenced by respiratory phase and load. Parameters are shown prior to separating by respiratory phase (black; combined) and during inspiratory (red), early expiratory (blue), and late expiratory (green) phases during eupneic breathing and mild and moderate resistance loads. (A) End 
diastolic pressure. (B) dP/dtmin. (C) Peak left ventricle pressure. (D) dP/dtmax. Data are presented as mean $\pm S D$. \#, significant interaction; $\uparrow$, main effect of respiratory load; $\neq$, main effect of respiratory phase; $a$, different from combined within a respiratory load; $b$, different from inspiration within a respiratory load; $c$, different from early expiration within a respiratory load; $d$, different from eupnea within a respiratory phase; $\mathrm{e}$, different from mild resistance within a respiratory phase; $p<0.05$ for all. All data were analyzed using a within-subject two-way ANOVA with main effects evaluated with one-way repeated measures ANOVAs and Sidak's correction, $\mathrm{n}=7$.

A

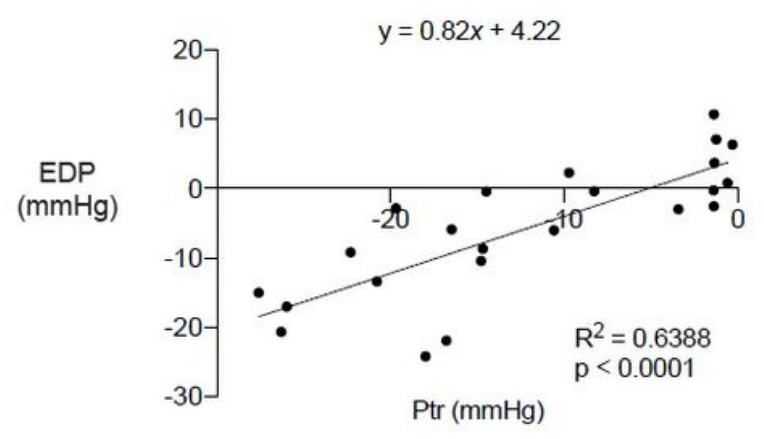

C

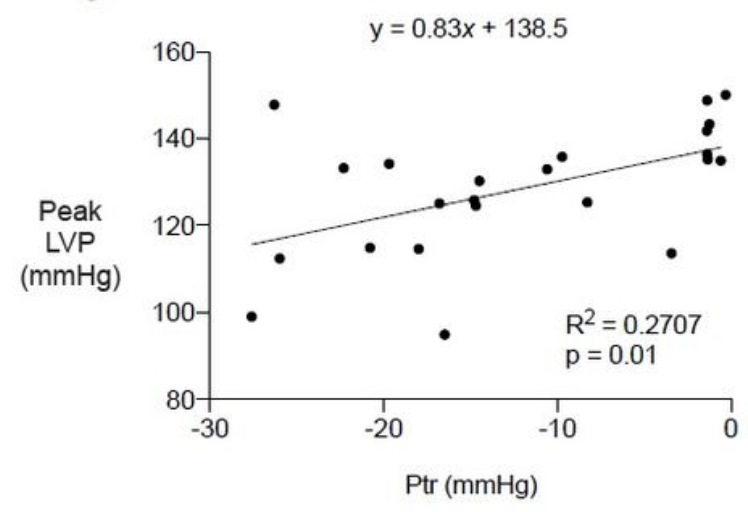

B

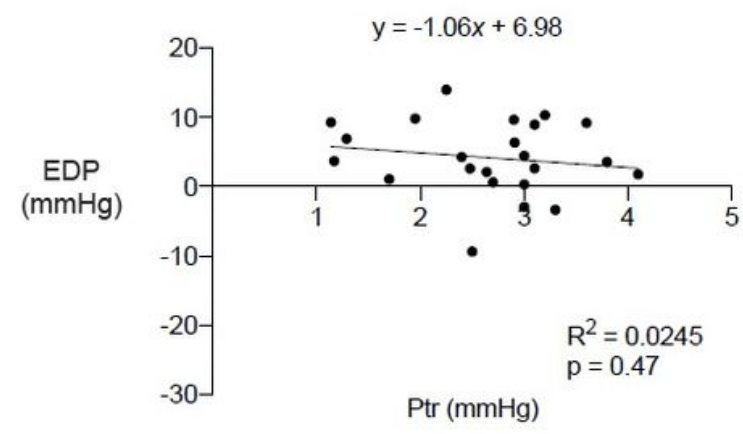

D

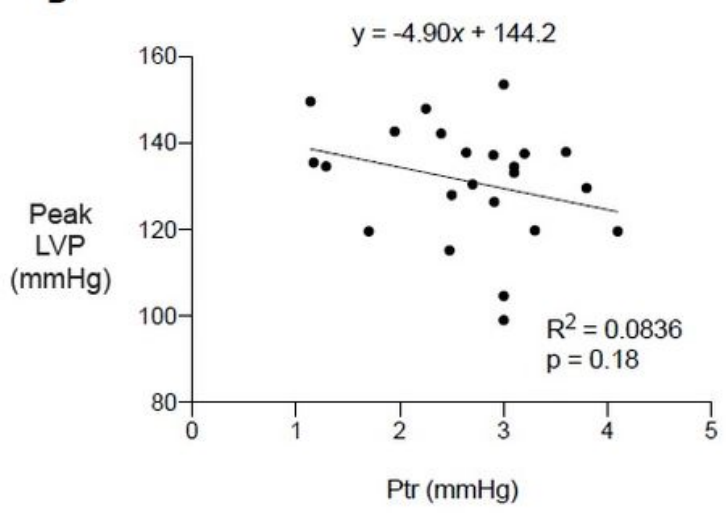

\section{Figure 4}

Diastolic and systolic pressures are predicted by inspiratory tracheal pressure. Linear regression of tracheal pressure versus end diastolic pressure (EDP) and peak left ventricle pressure (peak LVP). (A) EDP during inspiration. (B) EDP during late expiration. (C) Peak LVP during inspiration. (D) Peak LVP during late expiration. All data were analyzed by linear regression, $n=7$. 
v Combined - Inspiration a

A

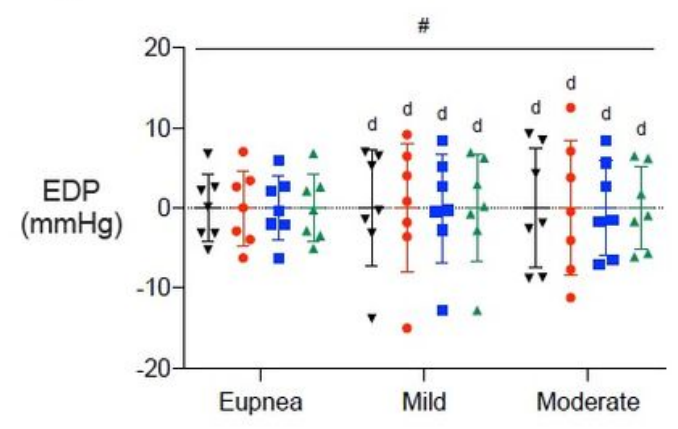

C

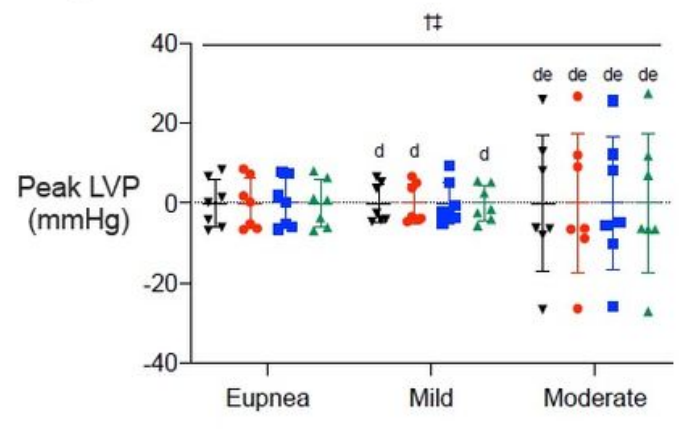

B

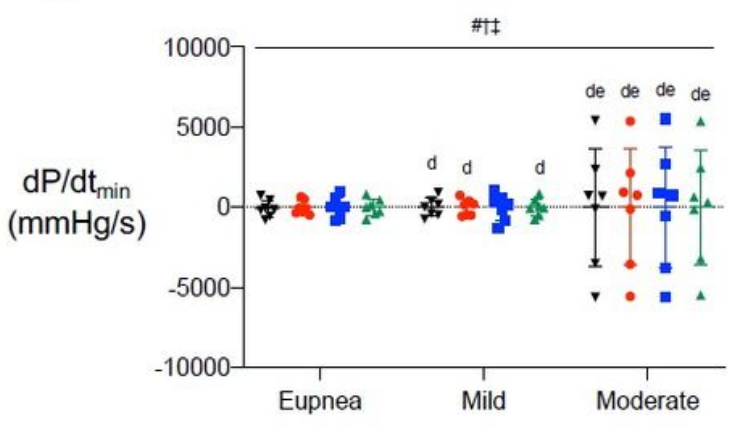

D

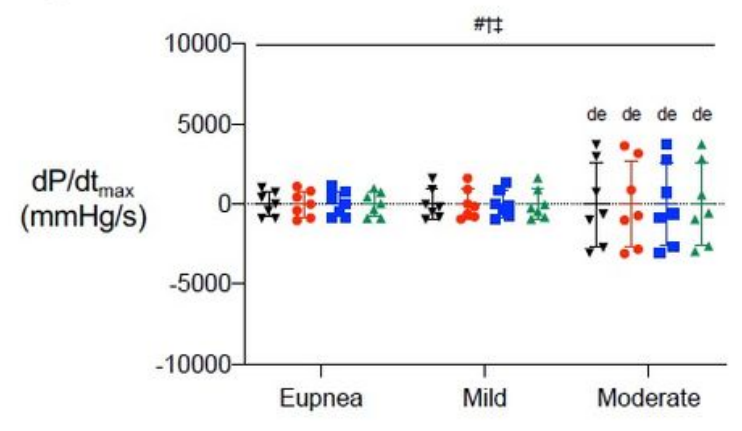

\section{Figure 5}

Respiratory resistance loading increases variance in cardiac parameters. Group means were subtracted from individual data points to set all means equal to zero and observe variance in each group.

Parameters are shown prior to separating by respiratory phase (black; combined) and during inspiratory (red), early expiratory (blue), and late expiratory (green) phases with eupneic breathing and mild and moderate resistance loads. (A) End diastolic pressure. (B) dP/dtmin. (C) Peak left ventricle pressure. (D) $\mathrm{dP} / \mathrm{dtmax}$. Data are presented as \pm SD. \#, significant interaction; $\dagger$, main effect of respiratory load; $\neq$, main effect of respiratory phase; $d$, different from eupnea within a respiratory phase; e, different from mild resistance within a respiratory phase; $p>0.05$ for all. All data were analyzed using a within-subject two-way ANOVA. Where Mauchly's test of sphericity was significant, one-tailed Pearson's correlation coefficients were determined. If normality was not assumed, Spearman's correlations were used, n=7. 


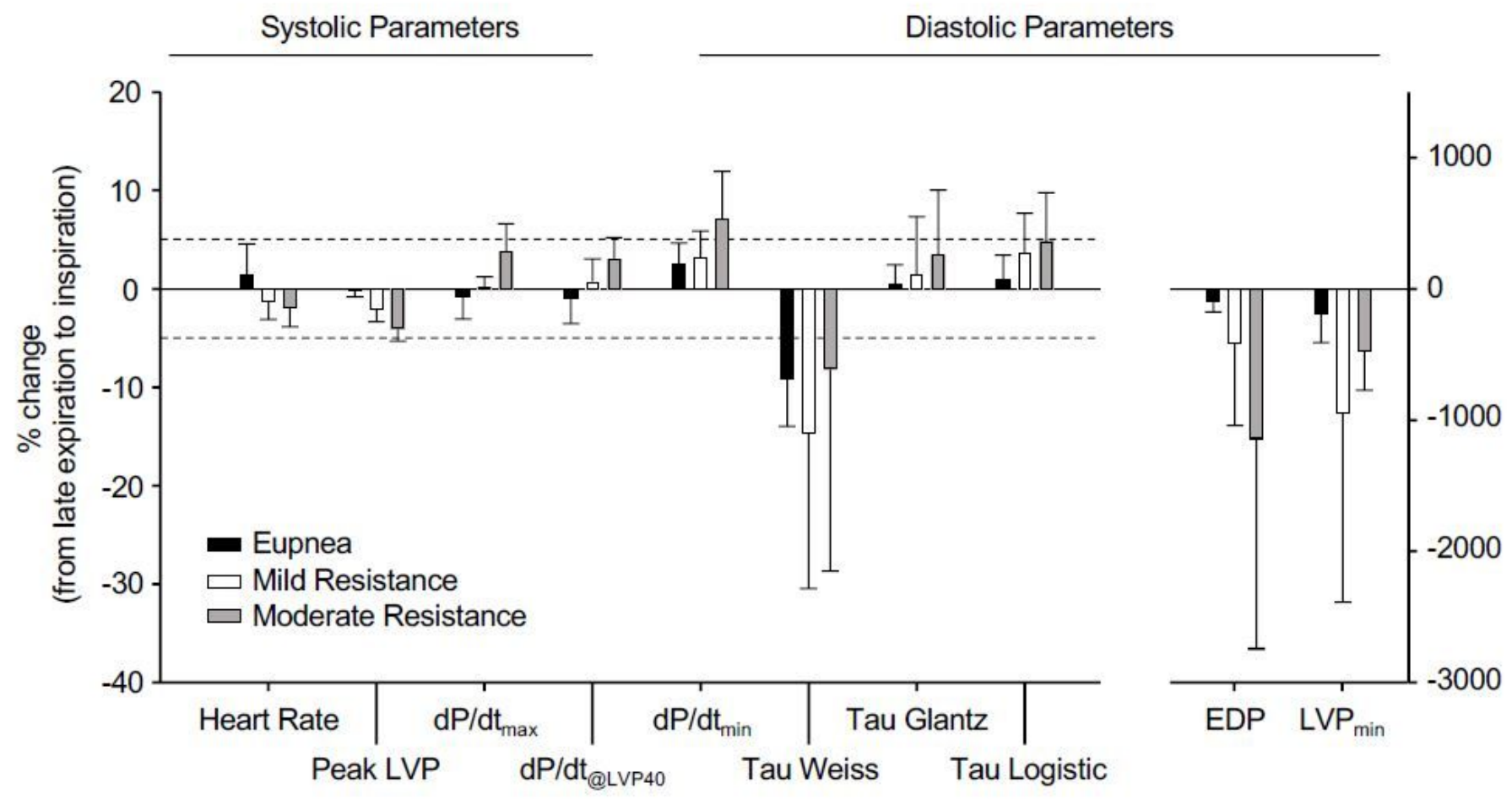

Figure 6

Diastolic parameters are more heavily influenced by inspiration than systolic parameters. Zero identifies the value specific to each parameter at the late expiratory phase of respiration. Vertical bars represent the percentage by which each parameter changes during inspiration from late expiration. Cardiac parameters are shown during eupnea (black), mild resistance (white), and moderate resistance (grey). Dotted horizontal lines are \pm 5 . Data are presented as mean $\pm S D, n=7$. 


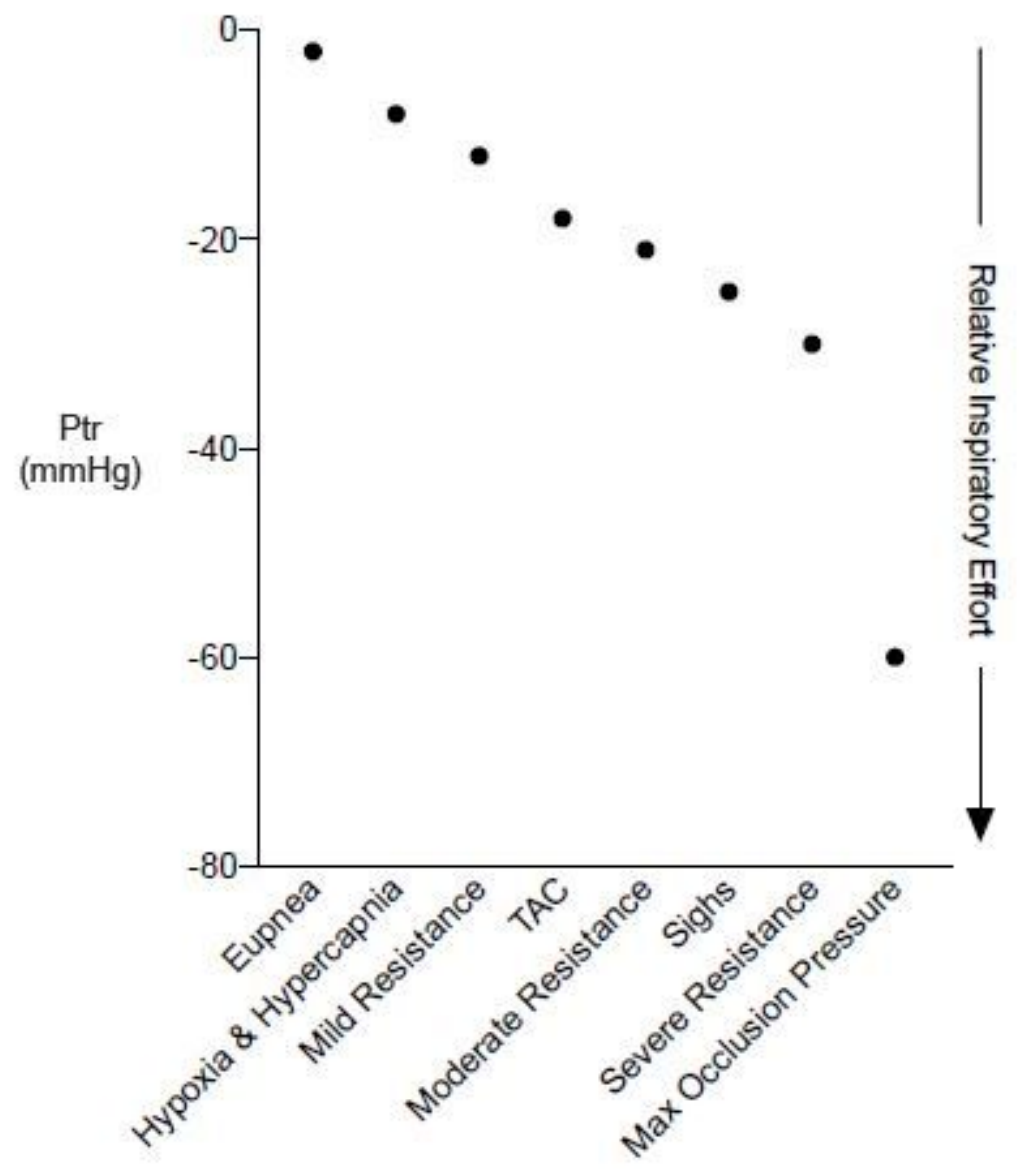

Figure 7

Summary figure. Schematic overview highlighting how inspiratory intrathoracic pressures produce immediate and delayed effects on ventricular pressures and volumes. RV, right ventricle; SV, stroke volume; EDV, end diastolic volume; EDP, end diastolic pressure; LVP, left ventricle pressure. Partially adapted from Amoore \& Santamore. 3 
Inspiration

$\downarrow$ Intrathoracic Pressure

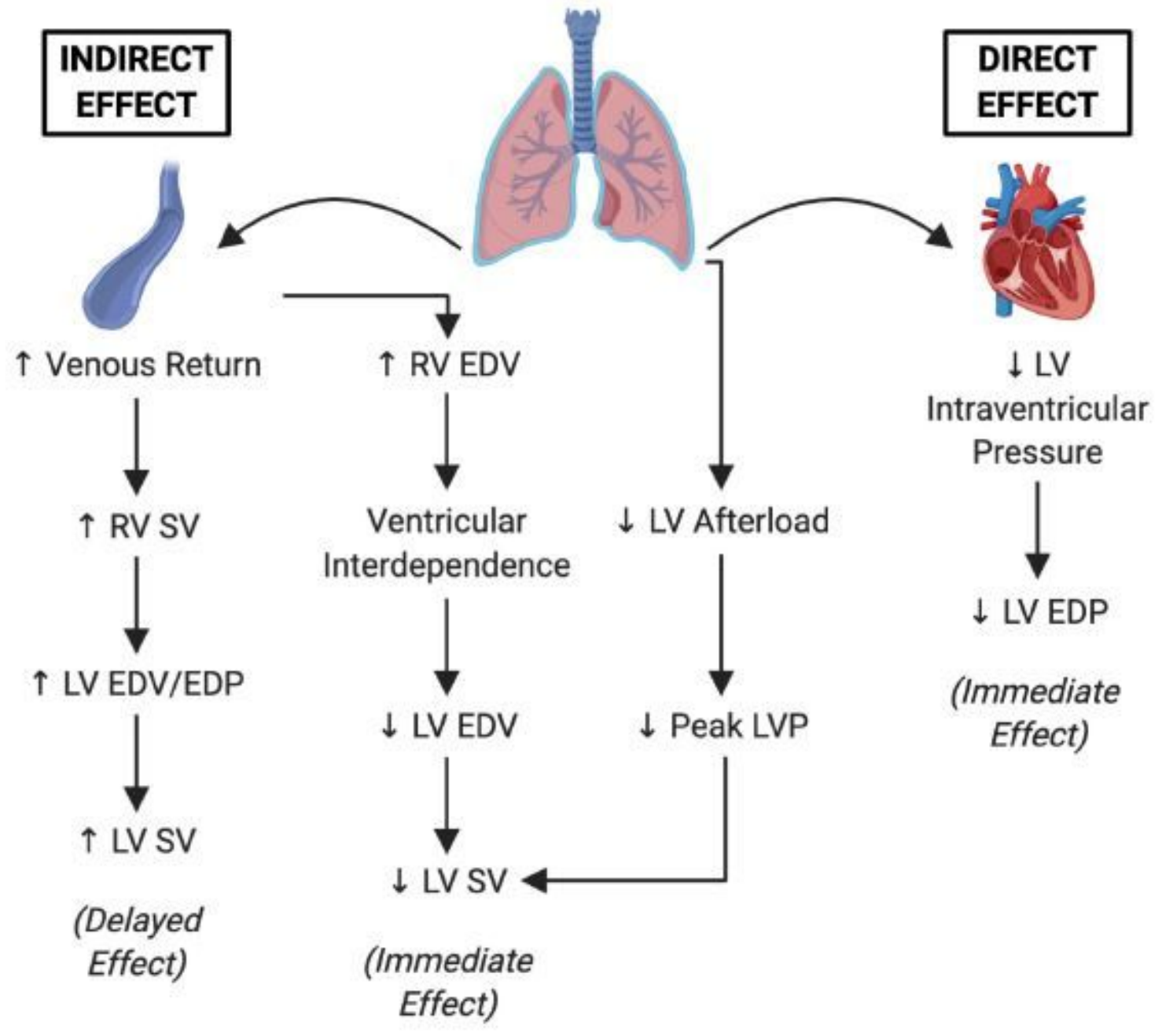

Figure 8

Inspiratory tracheal pressure for various respiratory efforts in rats. Representative tracheal pressure values expected across a range of cardiorespiratory conditions. Ptr, tracheal pressure; TAC, transverseaortic constriction to induce pressure-overload heart failure.

\section{Supplementary Files}

This is a list of supplementary files associated with this preprint. Click to download.

- FigureS1.pdf

- Figures2.pdf

- Tables1.pdf

- Tables2.pdf 
- Tables3.pdf

- Tables4.pdf

- Tables5.pdf

Page 22/22 